\title{
Creating the first Bose-Einstein condensate in space
}

M. D. Lachmann, H. Ahlers, D. Becker, S. T. Seidel, T. Wendrich, et al.

M. D. Lachmann, H. Ahlers, D. Becker, S. T. Seidel, T. Wendrich, E. M. Rasel, W. Ertmer, "Creating the first Bose-Einstein condensate in space," Proc. SPIE 10549, Complex Light and Optical Forces XII, 1054909 (22 February 2018); doi: 10.1117/12.2289686

SPIE. Event: SPIE OPTO, 2018, San Francisco, California, United States 


\title{
Creating the first Bose-Einstein Condensate in Space
}

\author{
M. D. Lachmann ${ }^{1}$, H. Ahlers ${ }^{1}$, D. Becker ${ }^{1}$, S. T. Seidel ${ }^{1}$, T. Wendrich ${ }^{1}$, E. M. Rasel ${ }^{1}$, W. \\ Ertmer $^{1}$ for the MAIUS-Team ${ }^{1,2,3,4,5,6,7,8}$ \\ ${ }^{1}$ Institut für Quantenoptik, Leibniz Universität Hannover, 30167 Hannover, Germany; ${ }^{2}$ \\ ZARM Bremen, 28359 Bremen, Germany; ${ }^{3}$ DLR-RY, 28359 Bremen, Germany; ${ }^{4}$ Institut für \\ Physik, Humboldt-Universität zu Berlin, 12489 Berlin, Germany; ${ }^{5}$ DLR-SC, 38108 \\ Braunschweig, Germany; ${ }^{6}$ Institut für Physik, Johannes Gutenberg-Universität Mainz, 55122 \\ Mainz, Germany; ${ }^{7}$ Institut für Laserphysik, Universität Hamburg, 22761 Hamburg, Germany; \\ ${ }^{8}$ Ferdinand-Braun-Institut, Leibniz Institut für Höchstfrequenztechnik, 12489 Berlin, \\ Germany;
}

\begin{abstract}
On $23^{\text {rd }}$ of January 2017 the first Bose-Einstein Condensate (BEC) in Space was created on-board the sounding rocket mission MAIUS-1. The successful launch marks a major advancement in the effort of performing matter wave interferometry with BECs on space vehicles.

Its high BEC-flux enables more than 100 experiments during flight, characterizing the creation of BECs in space, their free evolution, state preparation, and the creation of cold atoms in highly dynamic environments.

MAIUS-1 opens a new path towards space borne inertial sensing employing interferometers with high accuracy and sensitivity. Two follow-up missions will investigate dual-species interferometry. Recently several missions were proposed ranging from tests of the universality of free fall to gravimetry. Due to their small initial size and low expansion rates BECs are the ideal source for such an interferometric measurement. The findings of the mission will contribute to the NASA CAL project and BECCAL (NASA and DLR). This research is funded by DLR under grant 50WP1435.
\end{abstract}

Keywords: Atom interferometry in space, Bose-Einstein condensates

\section{INTRODUCTION}

The tests of fundamental principles of nature with increasing precision is a central goal of modern physics. One of these principles is the equivalence of inertial and gravitational mass. It states that all objects at the same position and free from other forces undergo the same acceleration through a gravitational field independent from their mass. It is one postulate of Einstein's general relativity and therefore of huge interest because some theoretical models permit violations $[1,2]$.

A simple classical test of this is to take two test objects with different masses, let them fall simultaneously and measure their accelerations. A more precise test taking into account the quantum nature of objects is the comparison of the free fall of two Bose-Einstein condensates (BEC), measuring their differential acceleration with atom interferometry [3]. Due to their slow expansion rates and mode properties Bose-Einstein condensates are a promising input state for this purpose $[4,5,6,7]$, because in order to increase the precision of such an interferometer the space-time-area enclosed in it has to be increased $[8,9]$. This can be achieved by performing the experiments in a weightless environment and then use longer free fall times of the cloud that scale squared with the sensitivity of the interferometric measurement [10]. The ideal platform for this are single satellites. They can provide microgravity times up to years and have a low noise environment compared to many laboratories on ground. But satellite missions have high financial as well as technological effort. All necessary methods need to be tested and qualified beforehand to minimize risks. 
In the German QUANTUS-collaboration (QUANTengase Unter Schwerelosigkeit) the feasibility to operate an atom interferometer in microgravity was demonstrated. For this the drop tower facilities in Bremen (Germany) were used to provide the microgravity environment by a free fall of the apparatus in a $120 \mathrm{~m}$ high evacuated tower. The creation of Bose-Einstein condensates [11] and their observation over one second as well as the realization of BEC-based atom interferometry in microgravity was shown for the first time [12].

As a next steps towards the transfer of such a system in space sounding rockets were planned as a platform for technology development and as a testbed of the methods needed for high precision atom interferometry in space [13]. The successful launch of the MAIUS-1 mission in January 2017 with the first BEC in space marks a major advancement towards a precise measurement of the equivalence principle with a space-born atom interferometer.

\section{DESCRIPTION OF THE APPARATUS}

For the MAIUS-1 mission the sounding rocket type was a VSB-30. It is a Brazilian rocket type with two boost stages that can carry scientific payloads of up to $450 \mathrm{~kg}$ [14] including the service module for communication to ground and a motor adapter. It performs a parabola flight and the reachable apogee depends on the total mass what limits the microgravity time, so the flight phase above $100 \mathrm{~km}$ height. Then it lands again using parachutes to avoid huge damages. Afterward the apparatus can be recovered.

The extreme conditions concerning accelerations and vibrations during launch and reentry result in high constraints in stability that were not given by common lab-based setups, so that additional developments and qualification on ground were necessary. This came together with the miniaturisation due to limited space on the vehicle [15].

The scientific payload MAIUS-A is able to create BECs of $10^{5}{ }^{87} \mathrm{Rb}$ atoms with a high repetition rate and to perform atom interferometry with them $[13,16]$. It consists of five modules. At the top of the scientific payload is the Physics Package with the vacuum chamber and the integrated three-layer atom chip. With the atom chip it is possible to create the necessary magnetic field for manipulation of the cloud with smaller volume and lower power consumption compared to coil setups. To get the high repetition rates a $2 \mathrm{D}^{+}$-MOT configuration is used in a second chamber to load the 3D-MOT. For detection there are two perpendicular camera axis, one using absorption detection and the other one using the fluorescence signal of the cloud. The ultra-high vacuum in the $10^{-11}$ mbar regime is provided by one ion getter pump and two titanium-sublimation pumps $[15,17]$. Around the vacuum chamber a three-layer magnetic shielding is attached to lower external fields by a factor of at least 1000 [18]. 

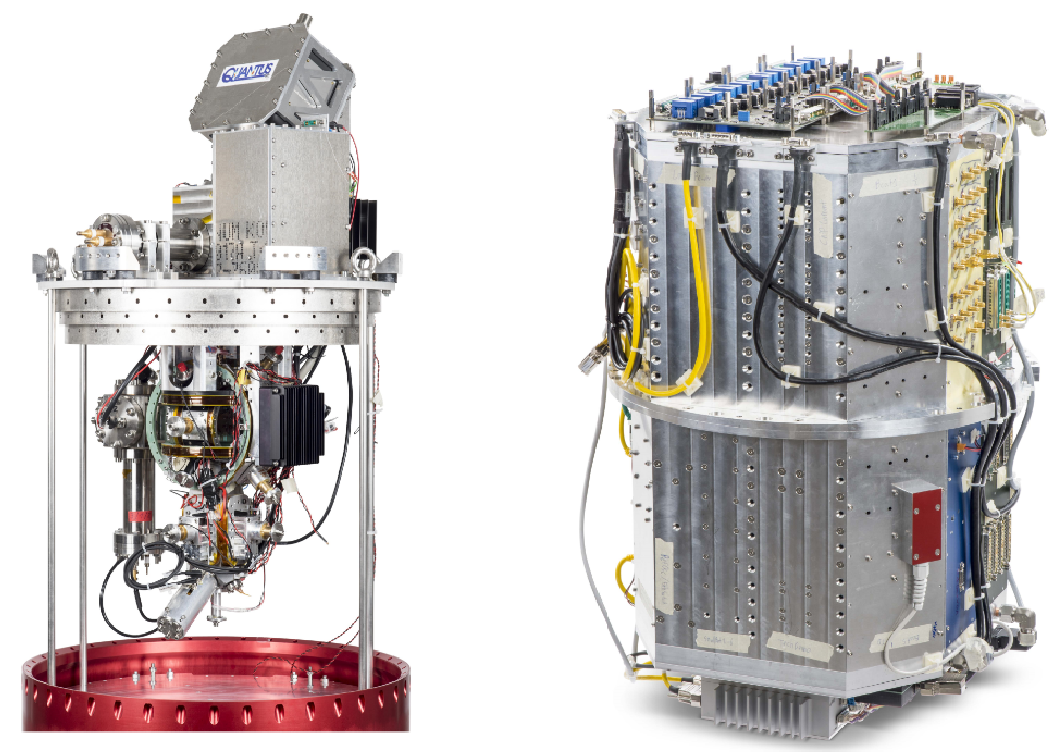

Figure 1. A picture of the Physics Package is shown on the left. It has a pumping stage on top and below the platform is the vacuum chamber with the integrated atom chip. The picture on the right shows the electronics module. The stacks are compact mounted in the housing that is used for mechanical and thermal stability.

Below the Physics Package the laser system is situated. It has a volume of 211 and a mass of $21 \mathrm{~kg}$ and contains all necessary lasers for the creation of a ${ }^{87} \mathrm{Rb} \mathrm{BEC}$ and to perform atom interferometry [19] and also two spare lasers and additional external cavity diode lasers (ECDL) as a technology demonstrator. Micro integrated distribution feedback (DFB) diode lasers and free space optical bench technology were combined and feature a master oscillator power amplifier (MOPA). Frequency stabilization of the master laser is done with a spectroscopy module using Doppler-free frequency modulation. The setup is mounted on Zerodur-boards to fulfil the mechanical and thermal stability requirements.

The electronics module consists of several stacks of custom-designed circuit boards to provide all the necessary functions for a BEC-based atom interferometer like temperature stabilization, current driver, frequency stabilization and pump control. The cards use a stackable connector for common signals like trigger, data and power as well as for analog connections between the cards resulting in a very compact module of only 100x100x17 mm2 per card. Each card is designed to support as many input and output channels as possible and is completely computer controlled.

Data storage is done at the on-board computer and one external device. The software for the flight was developed by the DLR-SC (Brunswig, Germany) and is optimized for autonomous operation.

For power supply of the apparatus lithium iron phosphate batteries were used. Arranged in different packs they can provide different voltages and supply the running experiment for around 45 minutes.

\section{MAIUS-1 MISSION}

After the successful qualification of the apparatus, including vibration, spin balancing and communication tests, the campaign took place at the ESRANGE (European space and Sounding rocket RANGE) in Sweden. The 
launch was at the $23^{\text {rd }}$ January 2017 at $3.30 \mathrm{am}$. The rocket reached an apogee of $243 \mathrm{~km}$ resulting in a microgravity time of 6 minutes.

Already during launch experiments with magneto-optical traps were performed. They were created, released and recaptured again for different time intervals to investigate the behaviour under these highly dynamic conditions.

Reaching the microgravity phase the rocket was stabilized concerning rotation and orientation. After an automatic new stabilization of the laser frequencies and a check of light power, vacuum pressure, temperatures and currents, the first Bose-Einstein condensate in space was created. Then further investigations in the phase transition were performed, followed by a characterization of the release out of the magnetic trap. The state preparation using an adiabatic rapid passage was autonomously optimized and a time of flight series was monitored to observe the free evolution of the condensate. Furthermore the ensemble was coherently split in two parts using Bragg diffraction beam splitters. Due to a modulation on the beams a spatial modulation on the density distribution of the atomic ensemble was observed.

$60 \mathrm{~km}$ away from the launch position the payload landed after 17 minutes of total flight time. Due to weather conditions the apparatus was recovered three days later. The temperatures in this time interval have been down to $-36^{\circ} \mathrm{C}$. After accurate investigations and some minor repairs the creation of BECs worked again. It was transported back to Hannover for further investigations necessary to analyse the flight results.

\section{OUTLOOK}

The knowledge gained with this mission helps now to develop new methods for future space missions with cold atoms. So are the lessons learned extremely important for the next two MAIUS missions which are planned to study dual-species atom interferometry in space. In addition to ${ }^{87} \mathrm{Rb}$ the new apparatus will contain ${ }^{41} \mathrm{~K}$ and perform experiments on mixtures in weightlessness environments, sequential and simultaneous dual-species interferometry and evolution of BECs on macroscopic time scales.

And the results of MAIUS-1 play a huge role for the BECCAL project with the goal to put a BEC-based multiuser facility on the International Space Station (ISS). These ambitious mission is a collaboration between the German Space agency (DLR) and the NASA (National Aeronautics and Space Administration).

\section{ACKNOWLEDGEMENTS}

QUANTUS and MAIUS are supported by the German Space Agency DLR with funds provided by the Federal Ministry of Economics and Technology (BMWi) under grant numbers DLR 50WM 1131-1137.

\section{REFERENCES}

[1] T. Damour, "Theoretical aspects of the equivalence principle," Classical and Quantum Gravity 29184001 (2012) 
[2] M. A. Hohensee, H. Müller, R. B. Wiringa, "Equivalence Principle and Bound Kinetic Energy,” Phys. Rev. Lett., 111, $151102(2013)$

[3] S. Dimopoulos, P.W. Graham, J.M. Hogan, and M.A. Kasevich, "Testing general relativity with atom interferometry,” Phys. Rev. Lett. 98, 111102 (2007)

[4] E.A. Cornell and C.E. Wieman, "Nobel Lecture: Bose-Einstein condensation in a dilute gas, the first 70 years and some recent experiments," Rev. Mod. Phys. 74, 875(2002)

[5] W. Ketterle, "Nobel Lecture: When atoms behave as waves: Bode-Einstein condensation and the atom laser," Rev. Mod. Phys. 74, 1131 (2002)

[6] Y. Torii, Y. Suzuki, M. Kozuma, T. Sugiura, T. Kuga, L. Deng, and E.W. Hagley, "Mach-Zehnder Bragg interferometer for a Bose-Einstein condensate," Phys. Rev. A 61, 041602 (2000)

[7] J.E. Simsarian, J. Denschlag, M. Edwards, C.W. Clark, L. Deng, E.W. Hagley, K. Helmerson, S.L. Rolston, and W.D. Phillips, "Imaging the Phase of an Evolving Bose-Einstein condensate Wave Function," Phys. Rev. Lett. 85, 2040 (2000)

[8] R. Geiger et al., "Detecting inertial effects with airborne matter-wave interferometry," Nat. Commun. 2, 474 (2011)

[9] J.E. Debs, P.A. Altin, T.H. Barter, D. Doring, G.R. Dennis, G. McDonald, R.P. Anderson, J.D. Close, and N.P. Robins, "Cold-atom Gravimetry with a Bose-Einstein condensate," Phys. Rev. A 84, 033610 (2011)

[10] H. Müller, S.-w. Chiow, S. Herrmann, S. Chu, and K.-Y. Chung, "Atom-Interferometry Tests of the Isotropy of Post-Newtonian Gravity,” Phys. Rev. Lett. 100, 031101 (2008)

[11] T. van Zoest et al., "Bose-Einstein Condensation in Microgravity,” Science 328, 1540 (2010)

[12] H. Müntinga et al., "Interferometry with Bose-Einstein Condensates in Microgravity," PRL 110, 093602 (2013)

[13] S. T. Seidel , "Eine Quelle für die Interferometrie mit Bose-Einstein Kondensaten auf Höhenforschungsraketen," Ph.D. thesis (Gottfried Wilhelm Leibniz Universität Hannover, 2014)

[14] A. Stamminger et al., "DLR's Mobile Rocket Base - Flight Tickets For Your Microgravity Experiments," 64th International Astronautical Congress Proceedings, International Astronautical Federation, Paris, 2013. ISSN 1995-6258.

[15] J. Grosse, "Thermal and Mechanical Design and Simulation for the first High Precision Quantum Optics Experiment on a Sounding Rocket," Ph.D. thesis (Universität Bremen, 2016)

[16] S. T. Seidel et al., "Atom Interferometry on Sounding Rockets," 22nd ESA Symposium on European Rocket and Balloon Programmes and Related Research, 730, 309 (2015)

[17] J. Grosse et al., "Design and qualification of an UHV system for operation on sounding rockets," J. Vac. Sci. Technol. A, Vol. 34, No. 3 (May/Jun 2016)

[18] A. Kubelka-Lange et al., "A three-layer magnetic shielding for the MAIUS-1 mission on a sounding rocket,” Rev. Sci. Instrum. 87, 063101 (2016)

[19] V. Schkolnik, O. Hellmig, A. Wenzlawski et al., "A compact and robust diode laser system for atom interferometry on a sounding rocket," Appl. Phys. B 122: 217 (2016) 